7th International Workshop on Astronomy and

Relativistic Astrophysics (IWARA 2016)

International Journal of Modern Physics: Conference Series

Vol. 45 (2017) 1760001 (8 pages)

(C) The Author(s)

DOI: $10.1142 / S 2010194517600011$

\title{
Walter Greiner: In Memoriam
}

\author{
César Zen Vasconcellos \\ Instituto de Fúsica \\ Universidade Federal do Rio Grande do Sul (UFRGS) \\ C. P. 15051, 91501-970 Porto Alegre, RS, Brazil \\ cesarzen@cesarzen.com \\ Helio T. Coelho* \\ Academia Brasileira de Ciências (ABC) \\ 20030-060, Rio de Janeiro, RJ, Brasil \\ Universidade Federal de Pernambuco (UFPE) \\ Cidade Universitária, 50670-901 Recife, PE, Brazil \\ heliotcoelho@terra.com.br \\ Peter Otto Hess \\ Instituto de Ciencias Nucleares \\ Universidad Nacional Autónoma de México (UNAM) \\ A.P. Postal 70-543, 04510 México, D.F., Mexico \\ hess@nucleares.unam.mx
}

Published 15 August 2017

\begin{abstract}
Walter Greiner (29 October 1935 - 6 October 2016) was a German theoretical physicist. His scientific research interests include the thematic areas of atomic physics, heavy ion physics, nuclear physics, elementary particle physics (particularly quantum electrodynamics and quantum chromodynamics). He is most known in Germany for his series of books in theoretical physics, but he is also well known around the world. Greiner was born on October 29, 1935, in Neuenbau, Sonnenberg, Germany. He studied physics at the University of Frankfurt (Goethe University in Frankfurt Am Main), receiving in this institution a BSci in physics and a Master's degree in 1960 with a thesis on plasmareactors, and a PhD in 1961 at the University of Freiburg under Hans Marshal, with a thesis on the nuclear polarization in $\mu$-mesic atoms. During the period of 1962 to 1964 he was assistant professor at the University of Maryland, followed by a position as research associate at the University of Freiburg, in 1964. Starting in 1965, he became a full professor at the Institute for Theoretical Physics at Goethe University until 2003. Greiner
\end{abstract}

*In 1972, H. T. Coelho was a post doctor at the University of Frankfurt and met Prof. Greiner which was already a famous professor there. A proficient scientific collaboration between them was then established.

This is an Open Access article published by World Scientific Publishing Company. It is distributed under the terms of the Creative Commons Attribution 4.0 (CC-BY) License. Further distribution of this work is permitted, provided the original work is properly cited. 
has been a visiting professor to many universities and laboratories, including Florida State University, the University of Virginia, the University of California, the University of Melbourne, Vanderbilt University, Yale University, Oak Ridge National Laboratory and Los Alamos National Laboratory. In 2003, with Wolf Singer, he was the founding Director of the Frankfurt Institute for Advanced Studies (FIAS), and gave lectures and seminars in elementary particle physics. He died on October 6, 2016 at the age of 80 .

Walter Greiner was an excellent teacher, researcher, friend. And he was a great supporter of the series of events known by the acronyms IWARA - International Workshop on Astronomy and Relativistic Astrophysics, STARS - Caribbean Symposium on Cosmology, Gravitation, Nuclear and Astroparticle Physics, and SMFNS - International Symposium on Strong Electromagnetic Fields and Neutron Stars. Walter Greiner left us. But his memory will remain always alive among us who have had the privilege of knowing him and enjoy his wisdom and joy of living.

\section{Walter Greiner: on the event horizon of a Black Hole}

Walter Greiner ${ }^{\mathrm{a}}$ made many important and ground breaking contributions in physics. They range from nuclear structure physics, heavy ion physics (the prediction of the island of stability, production of new super-heavy elements, investigations of the Quark Gluon Plasma,...) to atomic physics. Even how birds may orientate in the earth's magnetic field are on the list ${ }^{1}$ !

During the last eight years of his life he concentrated on an old hobby of his: How to avoid the formation of a black hole? It is intimately related to the elimination of the existence of the event horizon at the Schwarzschild radius for a non-rotating black hole (for a rotating black hole this event horizon is further in).

Many people always ask: Why to avoid the event horizon, it is only a coordinate singularity? There are always horizons lurking out there, as for example the one created by the receding of the galaxies (from a distance when they recede with a velocity larger than light) or for an accelerated observer.

But here the instinct and physical intuition of Walter set in: All horizons have a logical explanation to be there but not the one related to a black hole! He did question the situation where a part of space in the vicinity is completely locked out for a nearby observer, who can go around, come near to the black hole and has no possibility to access the space (except when he falls in, after which he will know what is happening inside, however, without a chance to communicate his findings to the outside). Also other strange things happen, for example most of the information gets lost (black holes don't have hairs).

The main reason for being suspicious is that all these phenomena are a consequence of a strong gravitational field. All other coordinate singularities do not have this origin. Furthermore, when large masses, and thus a strong gravitational field, is present: Why should the Theory of General Relativity still be working? It does not mean that the principles of this theory are not valid anymore, rather: It is possible

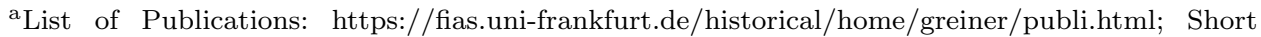
Biography: https://fias.uni-frankfurt.de/historical/home/greiner/bio.html; Honors and Positions: https://fias.uni-frankfurt.de/historical/home/greiner/honors.html. 
that the mass changes the surrounding space such that additional effects are coming in, not considered in the standard theory.

In the 70's he looked for possibilities to avoid that the radius of a collapsing star reaches the Schwarzschild radius, proposing the formation of strange stars or quark stars. All failed!

\section{The Pseudo-Complex General Relativity Theory}

In collaboration with P. O. Hess from the National University of Mexico, he addressed again this problem in a series of articles ${ }^{2-5}$ and demonstrated another tantalizing possibility, namely that mass might change the vacuum properties of the space nearby provoking the building up of dark energy, increasing toward smaller distances to the star. This is equivalent to a Casimir effect and the theory has a so-called pseudo-symplectic structure ${ }^{\mathrm{b}}$, a notion treated in one of the contributions of the present proceedings.

The theory allows to create stars of arbitrary size (see, for example 6,7 and predicts clear differences to the standard theory in the appearance of an accretion disk around a black hole, subject to soon verification by the Event Horizon Telescope. Actually $^{7}$ was his last contribution in this field.

The pseudo-complex theory, in its version up to 2015, are published in a book, ${ }^{8}$ the last one he wrote. There it is shown that the pseudo-complex theory has a much richer structure than finally used. For example it contains a minimal length which might be important when large masses collapse.

Unfortunately, he probably was not aware of the recent observation of gravitational waves and the interpretation of the source (see article by P. O. Hess in these proceedings) which would have excited him quite a lot.

Walter Greiner did have still a lot of plans, like to consider oscillating universes within the pseudo-complex theory and the role of the minimal length which is present in this theory. Unfortunately, these plans were interrupted by his death and somehow they will be finished by his collaborators. What we all are missing already is his insistence in pursuing with emphasis these goals and his ability to communicate the new findings to the world. Walter Greiner not only was an excellent physicists with an incomparable intuition but also a great science manager.

We all are missing you!

\footnotetext{
${ }^{\mathrm{b}}$ Einstein in the years 1945 and 1946 developed an algebraic extension of General Relativity Theory (GRT) by introducing complex-valued fields on real spacetime in order to apply Hermitian symmetry to GRT. Einstein introduced in his formalism a Hermitian metric whose real part is symmetric and describes the gravitational field while the imaginary part is antisymmetric and corresponds to the Maxwell field strengths. However, in this formulation the spacetime manifold remains real. Einstein soon recognized that this proposed unification does not satisfy the criteria that the metric tensor, $g_{\mu \nu}$, which characterizes the geometry of spacetime, should appear in GRT as a covariant entity with an underlying symmetry principle. In the year 2009, Peter O. Hess and Walter Greiner developed a new algebraic extension of the General Relativity Theory, named pc-GR, with pseudo-complex (pc) coordinates, which contains a minimal length and in addition requires the appearance of an energy-momentum tensor, related to vacuum fluctuations (dark energy), which are provoked by the presence of a central mass.
} 


\section{Prof. Dr. Dr. h.c. mult. Walter Greiner}

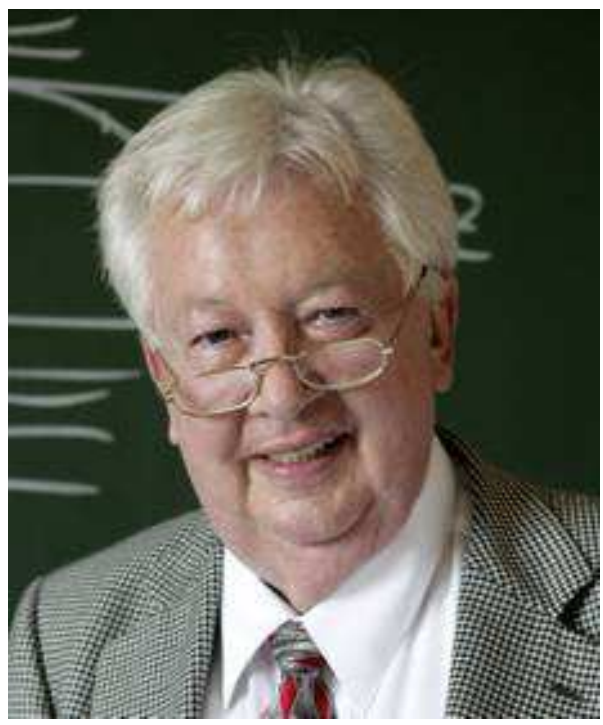

Fig. 1. Prof. Dr. Dr. h.c. mult. Walter Greiner.

- Professor Emeritus of Physics, Institute for Theoretical Physics, Johann Wolfgang Goethe-Universität - ITF/JWGU, Frankfurt Am Main, Germany;

- Founding Director, Frankfurt Institute for Advanced Studies - FIAS, Frankfurt Am Main, Germany.

\subsection{Scientific fields of research of Prof. Greiner}

- Nuclear Structure "Rotation-Vibration Model";

- Different Proton and Neutron Deformations in Nuclei;

- Dynamic Collective Model of Giant Resonances; The Spreading Width of Giant Resonances;

- Eigenchannel Theory of Nuclear Reactions;

- Generalized Collective Model (known as "Gneuß-Greiner"-Model or also the "Frankfurt" Collective Model);

- Prediction of Super-heavy Nuclei;

- The Two Center Shell Model;

- Theory of Nuclear Molecules;

- Prediction of New ("cluster") Radioactivities;

- Bimodal and Super-asymmetric Nuclear Fission;

- Heavy Ion Physics; 
- Quantum Electrodynamics (particularly of strong, overcritical fields "Decay of the Vacuum");

- Theory of Electronic Quasi-molecules in Heavy Ion Collisions;

- Structure of Super-heavy and Giant Nuclei;

- Shock Waves in Relativistic Heavy Ion Collisions: The Key Mechanism for Compressing and Heating Nuclear Matter;

- Nuclear Equation of State;

- Pion Bremsstrahlung;

- Phase Transitions of Nuclear Matter;

- Production of Clusters of Antimatter and Strange Matter from the Highly Correlated Vacuum (meson field theory);

- Extension of the Periodic System Into New Directions of Stangeness and Antimatter;

- Clusters of Matter and Antimatter - Super-dense Baryonic Clusters.

\subsection{Short biography of Prof. Greiner}

- 1956 - 1958 F.Sc. (Physics and Mathematics) - Johann Wolfgang Goethe University (JWGU), Frankfurt Am Main, Germany;

- Spring 1958 B.Sc. - Johann Wolfgang Goethe University (JWGU), Frankfurt Am Main, Germany;

- January 1960 M.Sc. TH Darmstadt, Thesis: "Plasma-reactors", Germany;

- 1960 - 1962 Research Associate to Prof. H. Marshall Freiburg/Brsg., Germany;

- Spring 1961 Ph.D. - Freiburg/Brsg. Thesis: "Nuclear polarization in $\mu$-mesic atoms", Germany;

- 1962 - 1964 Assistant Professor - University of Maryland (3 months Florida State University), USA;

- May 1964 Research Associate, University of Freiburg/Brsg., Germany;

- August 1964 Acceptance of a chair for theoretical physics - Johann Wolfgang Goethe University (JWGU), Frankfurt Am Main, Germany;

- Since Jan. 1965 Professor of Physics, Johann Wolfgang Goethe University (JWGU), Frankfurt Am Main, Germany; Director of the Institute of Theoretical Physics (1995), Frankfurt Am Main, Germany;

- Since 1964 Various offered professorships at Duke University, University of Virginia, Vanderbilt University, USA, declined;

- Since 1976 Permanent Consultant to Gesellschaft fr Schwerionenforschung (GSI) Darmstadt, Germany;

- Oct. 2001 - July 2002 Dean of the Physics Faculty of the Johann Wolfgang Goethe University (JWGU), Frankfurt Am Main, Germany;

- Since 2003 Founding Director and Member of the Management Board, Frankfurt Institute for Advanced Studies (FIAS), Frankfurt Am Main, Germany; 
- For many years he was a member of the scientific board (until to his death!) of the Joint Institute for Nuclear Research in Dubna, Moscow Oblast, Russia.

\subsection{Walter Greiner and the series of events IWARA (Brazil), STARS and SMFNS (Cuba)}

Walter Greiner was a great supporter of the series of events known by the acronym IWARA - International Workshop on Astronomy and Relativistic Astrophysics. The events of the IWARA series were held, until the year 2016, only in Brazil. In the year of 2018 the event will be held for the first time outside of Brazil, more precisely in Ollantaytambo, Peru, in the region of Machu Picchu. He would certainly be proud of this effective internationalization of the events of the IWARA series.

Walter was also the main driving force for the restructuring and strengthening of the series of events known presently by the acronyms STARS - Caribbean Symposium on Cosmology, Gravitation, Nuclear and Astroparticle Physics and SMFNS - International Symposium on Strong Electromagnetic Fields and Neutron Stars. One of us (CZV) recalls to this day the impact of the discussions held with Walter and Prof. Hugo Pérez-Rojas (ICIMAF, Cuba), in São Paulo, Maresias, Brazil, during the event IWARA 2011, in which Walter offered to us his insights about the future of these meetings in Cuba, and their role in opening up new research and job opportunities for young researchers and in strengthening research in particle physics, nuclear physics and astrophysics in the Caribbean region. His enthusiasm and power of persuasion in these discussions were overwhelmingly challenging.

Walter also liked to discuss the future of nuclear physics research, involving the discovery, exploration, and understanding of all forms of nuclear matter for a better understanding of how and why the fundamental particles of hadronic matter, quarks, and gluons fit together and interact. And create in this way different states of matter in the universe, including those states that are not found naturally.

Walter was particularly aware in addition to the future challenges of nuclear physics: providing the theoretical and experimental tools needed to produce scientific and technical advances that will lead to new knowledge, new skills and innovative discoveries and applications. In this context, we organized the themes of the first editions of the aforementioned IWARA series of events involving various topics such as: the existence and properties of nuclear material under extreme conditions, including the nuclear matter that existed at the beginning of the universe; exotic and excited states of quarks and gluons, including new tests of the Standard Model; physical limits of existence of complex systems of protons and neutrons; nuclear processes in supernovae, which synthesize the elements producing compact stars; nature and properties of neutrinos and neutrons and their role in the asymmetry between matter and the antimatter of the universe and related themes. 


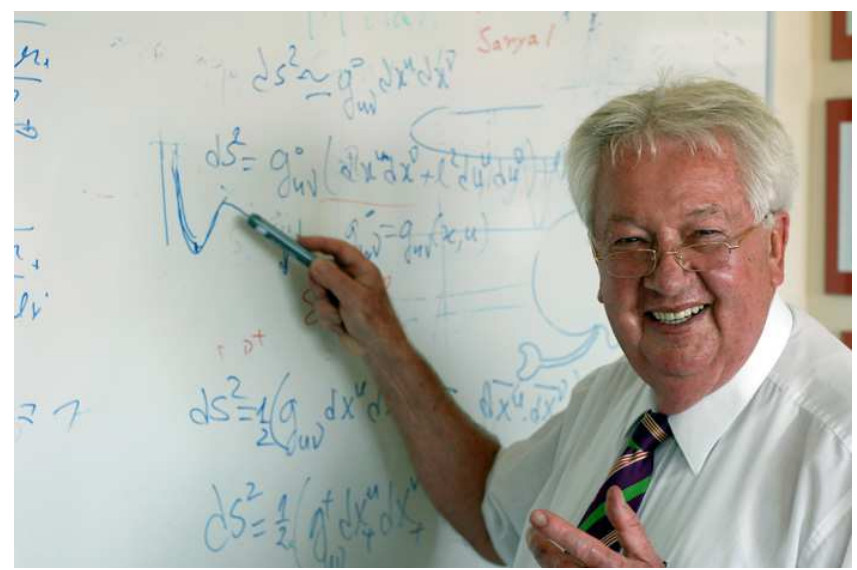

Fig. 2. Prof. Dr. Dr. h.c. mult. Walter Greiner.

The themes of the IWARA, STARS, and SMFNS series gradually them expanded with the inclusion of various areas of knowledge, involving today: new phenomena and new states of matter in the Universe, general relativity, gravitation, cosmology, heavy ion collisions and the formation of the quark-gluon plasma, white dwarfs, neutron stars and pulsars, black holes, gamma-ray emission in the Universe, high energy cosmic rays, gravitational waves, dark energy and dark matter, strange matter and strange stars, antimatter in the Universe, magnetic fields in the Universe, magnetic fields in compact stars and galaxies, ultra-magnetic magnetic fields in neutron star mergers, magnetic stars and magnetars, magnetic fields and the cosmic microwave background, and topics related to these.

The current denominations of the events in Cuba, STARS and SMFNS, was in reality Walter's idea. He imagined that with such denominations, those events could widen their scope. He also had in mind that these events would represent excellent opportunities for the establishment of new partnerships involving students and researchers from the most different regions of the planet and as motivation for attracting new students. Walter was always aware of these aspects and concerned with the development of the scientific spirit in the most remote regions of the planet. And his contribution to overcoming regional and institutional constraints has always been striking.

Walter went even further to help to materialize the events in Brazil and Cuba: he sent personal invitations for participation to many of the current members of the IWARA-STARS-SMFNS scientific community and he was responsible for literally opening the doors to important scientific journals so we could publish the proceedings of the IWARA, STARS and SMFNS series of events in their early stages.

These episodes undoubtedly demonstrate the unselfish and generous manner in which Walter stood and related to his students, colleagues, and friends. His memory for this will always be alive between us. 


\section{References}

1. I.A. Solov'yov, PhD thesis Magnetoreception Mechanisms in Birds - towards the discovery of the sixth sense, Univeristy of Frankfurt, Germany (2007) (D30) and references therein.

2. Peter O. Hess and Walter Greiner, Int. J. Mod. Phys. E 18, 51 (2009).

3. G. Caspar, T. Schönenbach, P. O. Hess, M. Schäfer and W. Greiner, Int. J. Mod. Phys. E 21, 1250015 (2012).

4. T. Schönenbach, G. Caspar, P. O. Hess, T. Boller, A. Müller, M. Schäfer and W. Greiner, MNRAS 430, 2999 (2013).

5. T. Schönenbach, G. Caspar, P. O. Hess, T. Boller, A. Müller, M. Schäfer and W. Greiner, MNRAS 442, 121 (2014).

6. I. Rodríguez, P. O. Hess, S. Schramm and W. Greiner, J. Phys. G 41, 105201 (2014).

7. G. Caspar, I. Rodríguez, P. O. Hess and W. Greiner, Int. J. Mod. Phys. E 25, 1650027 (2016).

8. P. O. Hess, M. Schäfer and W. Greiner;Pseudo-Complex General Relativity, (Springer, Heidelberg, 2015); ISBN 978-3-319-25060-1, DOI 10.1007/978-3- 319-25061-8. 